\title{
Estado nutricional e risco de quedas em idosos hospitalizados
}

\section{Nutritional status and risk of fall in hospitalized elderly}

\author{
Maria Luiza Freitas Annes' ${ }^{1}$ Daniela de Souza Motta Marchi², Janete de Souza Urbanetto², \\ Raquel Milani El Kik ${ }^{4}$
}

1 Nutricionista. Mestranda no Programa de Pós-Graduação em Gerontologia Biomédica do Instituto de Geriatria e Gerontologia da Pontifícia Universidade Católica do Rio Grande do Sul (PUCRS),Porto Alegre, RS, Brasil._maluannes@hotmail.com>

${ }^{2}$ Nutricionista. Especialista em Saúde do Idoso. Preceptora do Programa de Residência Multiprofissional da Pontifícia Universidade Católica do Rio Grande do Sul (PUCRS), Porto Alegre, RS, Brasil.<mottadany@ig.com.br>

${ }_{3}^{3}$ Enfermeira. Doutora em Ciências da Saúde pela Pontifícia Universidade Católica do Rio Grande do Sul. Professora do Curso de Graduação em Enfermagem da Pontifícia Universidade Católica do Rio Grande do Sul (PUCRS), Porto Alegre, RS, Brasil. <jurbanetto@pucrs.br>

${ }^{4}$ Nutricionista. Doutoranda no Programa de Pós-Graduação em Gerontologia Biomédica do Instituto de Geriatria e Gerontologia da Pontifícia Universidade Católica do Rio Grande do Sul (PUCRS), Professora Adjunta do Curso de Graduação em Nutrição da PUCRS, Porto Alegre, RS, Brasil. < raquel.elkik@pucrs.br>

\section{ARTICLE INFO}

\section{Article history}

Received: 18/12/2016

Accepted: 18/03/2017

\section{Correspondent Author}

Maria Luiza Freitas Annes

Rua Carlos Silveira Pacheco Martins,

$55 / 704 \mathrm{G}$

91350-300 Porto Alegre, RS, Brasil

<maluannes@hotmail.com>

\section{(c) 2016 All rights reserved}

\section{Editors}

Alfredo Cataldo Neto

Paula Engroff

\begin{abstract}
RESUMO
Objetivo deste estudo é verificar a relação entre o estado nutricional e o risco de quedas em idosos hospitalizados. Trata-se de um estudo transversal, com amostra composta por idosos ( $\geq 60$ anos), admitidos na unidade de internação geriátrica, de um hospital de ensino, no período de maio de 2014 a setembro de 2015. As variáveis avaliadas foram dados demográficos (sexo e idade), estado nutricional pela Mini Avaliação Nutricional ${ }^{\circledR}$ e risco de quedas através da Morse Fall Scale. Participaram 96 pacientes, com média de

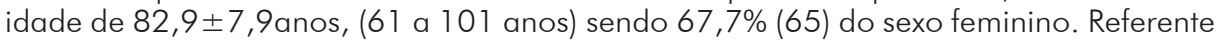
à Mini Avaliação Nutricional ${ }^{\circledR}$, 53,2\% (41) encontravam-se desnutridos, 35, $1 \%$ (27) com risco de desnutrição e 11,7\% (9) com estado nutricional normal. Quanto à Morse Fall Scale, 68,8\% (66) dos pacientes encontravam-se com risco elevado de quedas, $26 \%$ (25) com risco moderado e 5,2\% (5) com risco baixo. Não foi identificada associação entre o estado nutricional e o risco de quedas $(p=0,339)$, embora observou-se que $59,6 \%$ dos idosos desnutridos tinham risco elevado de quedas e $47,6 \%$ daqueles com risco nutricional tinham risco moderado de quedas. Os idosos apresentaram risco elevado de quedas, além do alto índice de desnutrição e risco de desnutrição. Não houve associação entre estado nutricional e risco de quedas.
\end{abstract}

PalAVRAS-ChaVe: Idoso; Nutrição do idoso; Quedas; Estado nutricional.

\begin{abstract}
The aim of this study is to investigate the relationship between nutritional status and the risk of falls in hospitalized elderly. This is a cross-sectional study with a sample of elderly ( $\geq 60$ years), admitted to the geriatric inpatient unit of a teaching hospital, from May 2014 to September 2015 . The variables were demographic data (gender and age), nutritional status the Mini Nutritional Assessment ${ }^{\circledR}$ and risk of fall through the Morse Fall Scale. There were 96 inpatients in this study, the average age was between $82.9 \pm 7.9$ years (61 to 101 years), being $67.7 \%(65)$ from the female gender. According to the Mini Nutritional Assessment ${ }^{\circledR}$, 53.2\% (41) were malnourished, 35.1\% (27) were at risk and $11.7 \%$ (9) were eutrophic. According to the Morse Fall Scale, 68.8\% (66) of the patients had high risk of falling, $26 \%$ (25) had moderate risk and $5.2 \%$ (5) were at low risk. There was no association between the nutritional status and the risk of fall, although it was observed that $59.6 \%$ of the malnourished elderly had high risk of falling and $47.6 \%$ at nutritional risk had also medium risk of falling. The elderly presented high risk of falls, besides high incidence of malnourishment. Despite that, there was no association between nutritional status and risk of fall.

KEYwORDS: Aged; Elderly nutrition; Accidental falls; Nutritional status.
\end{abstract}




\section{INTRODUÇÃO}

Os idosos são hospitalizados com uma frequência três vezes maior que as pessoas mais jovens, ${ }^{1}$ assim como apresentam permanência hospitalar mais prolongada do que pacientes de outras faixas etárias. 2,3 Em 2009, foram registradas mais de 2 milhões de internações hospitalares de pacientes com idade $\geq 60$ anos no sistema público de saúde brasileiro, que correspondem a $21 \%$ das admissões hospitalares no período. ${ }^{4}$

No que diz respeito à saúde, a problemática tende a ser a mesma que se verifica nos países desenvolvidos (doenças crônicas requerendo cuidados continuados e onerosos), agravada pelo fato de persistirem problemas como desnutrição e doenças infecciosas. ${ }^{5}$ A desnutrição é a sexta causa de internação hospitalar mais frequente entre os homens com idade $\geq 80$ anos, com uma taxa de 5,3\%. ${ }^{6}$ A desnutrição é um problema comum no idoso, sendo considerado o distúrbio mais importante nesta fase da vida, contribuindo no aumento da mortalidade, da susceptibilidade às infecções e na redução da qualidade de vida. A desnutrição é comum em idosos, muitas vezes acelera a perda de massa muscular e diminui a força muscular ou a capacidade de manter o equilíbrio. ${ }^{7} \mathrm{~A}$ desnutrição muitas vezes se desenvolve discretamente e pode não ser notada, a menos que seja rastreada o mais precoce possível. Assim, a triagem nutricional é um passo necessário para identificar aqueles que estão em risco de desnutrição para a intervenção precoce. ${ }^{8}$ A prevalência de desnutrição em idosos hospitalizados atendidos pelo Sistema Único de Saúde foi de 52,8\%, conforme o estudo multicêntrico IBRANUTRI. ${ }^{9}$

O propósito da avaliação nutricional, além de identificar o diagnóstico da desnutrição, é apontar pacientes que apresentam um maior risco de sofrer complicações associadas ao estado nutricional durante sua internação. Desta forma, a avaliação nutricional é considerada um instrumento tanto prognóstico como diagnóstico muito importante na admissão do paciente no hospital. ${ }^{10}$

Segundo a Organização Mundial da Saúde, as quedas são definidas como o evento em que a pessoa "inadvertidamente cai ao solo ou níveis inferiores, excluindo mudança intencional da posição para repouso na mobília, parede ou outros objetos". ${ }^{11}$

Neste contexto, as quedas também são bastante comuns na população idosa e estão associadas à desnutrição, podendo mesmo ser causa ou consequência desta. ${ }^{12}$ As quedas podem ocorrer a partir da perda da força muscular e da perda de funcionalidade nos idosos. No Brasil, 30\% dos idosos caem pelo menos uma vez por ano, tendo relação causal com $12 \%$ dos óbitos nesta população. Além disso, suas conseqüências incluem, dentre outras, lesões físicas, declínio funcional, medo de cair e restrição de atividades. As quedas ocorrem mais frequentemente em idosos hospitalizados, quando comparados com os que vivem na comunidade. Estimase que um em cada cinco irá cair durante o período de internação. A associação entre o ambiente hospitalar e o aumento do risco de quedas ocorre, muitas vezes, pelo fato de que, na admissão, os idosos acumulam fatores de risco adicionais para quedas, incluindo o ambiente novo e estranho, no qual são pouco conhecidos os fatores de risco externos para quedas e, freqüentemente, o desenvolvimento de confusões e doenças agudas. ${ }^{13}$

A Mini Avaliação Nutricional (MNA) é uma ferramenta simples, não invasiva e eficiente feita para avaliar/rastrear o risco de desnutrição em idosos. É composta por dezoito itens, que contem avaliação antropométrica, dietética, global e auto avaliação referente a aspectos nutricionais. ${ }^{14,15}$

Desta forma o presente estudo teve como objetivo analisar a associação entre o estado nutricional e o risco de quedas em idosos hospitalizados.

\section{MÉTODOS}

Trata-se de um estudo transversal, com amostra composta por idosos ( $\geq 60$ anos), admitidos na unidade de internação geriátrica, de um hospital de ensino, no período de maio de 2014 a setembro de 2015. Foram considerados os pacientes que podiam responder as questões, e aqueles que não conseguiam, os cuidadores puderam responder. As variáveis avaliadas foram dados demográficos (sexo e idade), estado nutricional pela Mini Avaliação Nutricional ${ }^{\circledR}\left(\mathrm{MNA}^{\circledR}\right)$ e risco de quedas através da versão brasileira da Morse Fall Scale (MFS) ${ }^{16}$

A MNA ${ }^{\circledR}$ foi aplicada de forma global em todos os idosos hospitalizados. Trata-se de um questionário, que engloba um total de 18 itens, que inclui antropometria, avaliação dietética, avaliação clínica global e autopercepção de saúde e do estado nutricional. Os resultados obtidos classificam os idosos em: desnutridos $(<17$ pontos), com risco de desnutrição (17 a 23,5 pontos) e com estado nutricional normal ( 24 a 30 pontos). ${ }^{17}$

A MFS é um instrumento de rápida e fácil aplicação que permite avaliar a probabilidade de quedas. É amplamente utilizada em ambiente hospitalar, em doentes na fase aguda e em centros de internamento de doentes crônicos. Esta escala encontra-se organizada em seis parâmetros, que originam uma pontuação, que varia entre 0 e 125 pontos, sendo eles: história de queda nos últimos três meses, diagnóstico secundário, ajuda na mobilização, terapia endovenosa, marcha e estado mental. Depois de somada a pontuação obtida, em cada uma das seis variáveis, o total de pontos alcançado 
permite identificar o nível de risco, sendo risco baixo de quedas ( 0 a 24 pontos), risco moderado de quedas ( 25 a 44 pontos) e elevado risco de quedas ( $\geq 45$ pontos). ${ }^{18}$

Os dados foram armazenados em banco de dados Excel e analisados através do pacote estatístico SPSS 17.0 (SPSS Inc. Chicago IL, USA). A análise estatística foi descritiva, a partir das medidas de tendência central e variabilidade, tabelas de frequência simples e cruzadas. A comparação entre estado nutricional e risco de quedas foi realizada através do Teste Exato de Fisher. Este estudo é secundário a dois projetos aprovados pelo Comitê de Ética em Pesquisa da PUCRS, sendo "Perfil nutricional de pacientes do Hospital São Lucas da PUCRS” (parecer 10/05006) e "Associação entre o risco e a ocorrência de quedas de pacientes hospitalizados" (Parecer 1272/09).

\section{RESULTADOS}

Participaram 96 pacientes, com média de idade de $82,9 \pm 7,9$ anos, com intervalo de 61 a 101 anos, sendo $67,7 \%$ (65) do sexo feminino, a maioria foi classificada com desnutrição $(53,2 \%)$ e com risco elevado de quedas $(68,8 \%)$, conforme observa-se na Tabela 1 . Referente à $\mathrm{MNA}^{\circledR}$ foram avaliados 77 pacientes, pois 19 encontravam-se sem condições de responder à avaliação e estavam sem acompanhante.

Tabela 1. Classificação de idosos hospitalizados de acordo com a Mini Avaliação Nutricional ${ }^{\circledR}$ e a Morse Fall Scale

\begin{tabular}{lc}
\multicolumn{1}{c}{ Variáveis } & $\begin{array}{c}\text { Frequência } \\
\text { N (\%) }\end{array}$ \\
\hline Mini Avaliação Nutricional ${ }^{\circledR}$ & \\
Desnutrição & $41(53,2)$ \\
Risco de desnutrição & $27(35,1)$ \\
Sem risco de desnutrição & $9(11,7)$ \\
Morse Fall Scale & \\
Risco elevado de quedas & $66(68,8)$ \\
Risco moderado de quedas & $25(26)$ \\
Risco baixo de quedas & $5(5,2)$ \\
\hline
\end{tabular}

A associação entre o estado nutricional e o risco de quedas não foi estatisticamente significativa, conforme pode ser observado na Tabela 2. Porém, pode-se observar que, dos pacientes que apresentavam desnutrição, 59,6\% (31) apresentavam risco elevado de quedas.

\section{DISCUSSÃO}

De acordo com o presente estudo, verificou-se, entre os idosos, maior frequência de risco de desnutrição e desnutrição, assim como a presença de alto risco de quedas, embora não tenha sido verificada associação entre as variáveis.

A alta frequência de desnutrição encontrada no estudo vem sendo descrita na literatura. Silva et al., 2014, avaliaram 167 idosos institucionalizados, em Recife, através da $\mathrm{MNA}^{\circledR}$, onde $52,1 \%$ estavam em risco nutricional. ${ }^{18}$ No estudo de Duarte et al., 2014, com 100 idosos hospitalizados, no interior do Rio Grande do Sul, 49\% dos idosos apresentaram risco de desnutrição. ${ }^{19} \mathrm{Um}$ estudo desenvolvido com 52 idosos hospitalizados, em Minas Gerais, obteve 38\% de idosos com desnutrição. ${ }^{20}$

A frequência elevada de idosos com desnutrição e risco de desnutrição é comum em várias populações, em especial os idosos internados ou institucionalizados, isso se deve as patologias, que muitas vezes levam à perda ponderal pela redução da ingestão de alimentos. ${ }^{20}$

Resultados semelhantes aos do presente estudo, em relação ao risco de quedas, avaliados pela MSF, foram observados por Rocha et al. ${ }^{21}$ (2013), que avaliaram 84 adultos e idosos hospitalizados, e verificaram que $45,2 \%$ (38) dos pacientes estavam com risco elevado $32,1 \%$ (27) com risco moderado e $22,6 \%$ (19) com risco baixo de queda. ${ }^{21}$ Bailey et al..$^{22} \mathrm{em}$ um estudo em clínica geriátrica canadense, verificou que $2,2 \%$ dos idosos tinham risco baixo, $21,2 \%$ risco moderado e $76,6 \%$ possuíam escore de risco elevado de quedas. ${ }^{22}$

Tabela 2. Associação entre o estado nutricional e o risco de quedas em idosos hospitalizados

\begin{tabular}{|c|c|c|c|c|}
\hline \multirow[b]{2}{*}{ Morse Fall Scale } & \multicolumn{3}{|c|}{ Mini Avaliação Nutricional } & \multirow[b]{2}{*}{$\mathbf{P}^{*}$} \\
\hline & $\begin{array}{c}\text { Desnutrido } \\
\text { N (\%) }\end{array}$ & $\begin{array}{c}\text { Em risco de } \\
\text { desnutrição } \\
\text { N (\%) }\end{array}$ & $\begin{array}{c}\text { Sem risco de } \\
\text { desnutrição } \\
\text { N (\%) }\end{array}$ & \\
\hline Risco elevado de quedas & $31(59,6)$ & $16(30,8)$ & $5(9,6)$ & \multirow{3}{*}{$<0,339$} \\
\hline Risco moderado de quedas & $8(38,1)$ & $10(47,6)$ & $3(14,3)$ & \\
\hline Risco baixo de quedas & $2(50)$ & $1(25)$ & $1(25)$ & \\
\hline
\end{tabular}

* Teste Exato de Fisher. 
Em um estudo com mais de 4 mil participantes em Taiwan de moradias comunitárias, verificou-se através da MNA ${ }^{\circledR}$ que da amostra total 1,3\% apresentavam desnutrição e 9,9\% risco de desnutrição e que a desnutrição foi um dos fatores de risco independentes para queda. ${ }^{23}$

Um estudo realizado em 81 instituições de longa permanência na Holanda, avaliou o estado nutricional e a ocorrência de quedas nos últimos 30 dias da avaliação em 6701 participantes. Verificou-se que $31,2 \%$ dos idosos avaliados pelo IMC, perda de peso e alteração na ingestão alimentar apresentava risco de desnutrição e $28,8 \%$ desnutrição e observaram que os pacientes desnutridos tinham mais chances de cair dos que aqueles sem risco nutricional. ${ }^{24}$

Não foram identificados estudos que analisaram a associação entre a classificação do estado nutricional pela $\mathrm{MNA}^{\circledR}$ e o risco de quedas pela MFS.

\section{CONCLUSÃO}

Os idosos hospitalizados apresentaram risco elevado de quedas e alto índice de desnutrição e risco de desnutrição. Apesar disso, não foi verificada associação entre o estado nutricional e o risco de quedas.

\section{REFERÊNCIAS}

1. Landefeld CS. Improving health care for older persons. Ann Intern Med. 2003;139(5):421-4.

2. Cavalcanti MGPH, Saad PM. Os idosos no contexto da Saúde Pública. In: Fundação Sistema Estadual de Análise de Dados, organizador. O idoso na Grande São Paulo, São Paulo: Fundação Sistema Estadual de Análise de Dados; 1990. p. 181-205.

3. Veras RP, Coutinho E, Coeli CM. Transtornos mentais em idosos: a contribuição da epidemiologia. In: Veras RP, organizador. Terceira idade: desafios para o terceiro milênio. Rio de Janeiro: Relume-Dumará/Universidade Aberta da Terceira Idade/Universidade do Estado do Rio de Janeiro; 1997. p. 15-40.

4. Brasil. DATASUS. Informações de Saúde Demográficas e Socioeconômicas [cited 2015 Out 26]. Available from: http://www2.datasus.gov.br/DATASUS/index.php?area =02

5. Instituto de Estudos de Saúde Suplementar. Envelhecimento populacional e os desafios para o sistema de saúde brasileiro [recurso eletrônico] / Instituto de Estudos de Saúde Suplementar - São Paulo: IESS [org]; 2013

6. Instituto Brasileiro de Geografia e Estatística - IBGE Indicadores Sociodemográficos e de Saúde no Brasil 2009, Rio de Janeiro; 2009.

7. Vanltallie T.B. A fragilidade em idosos: contribuições da sarcopenia e exaustão de proteína visceral Metabolismo. 2003;52:22-6.
8. Tsai AC, Lai M-Y. Mini Nutritional Assessment and shortform Mini Nutritional Assessment can predict the future risk of falling in older adults e Results of a national cohort study. Clinical Nutrition. 2014;33(5):844, 849.

9. Waitzberg DL, Caiaffa WT, Correia MITD. Hospital Malnutrition: The Brazilian National Survey (IBRANUTRI): a study of 4000 patients. Nutrition. 2001;17(7/8):573-80.

10. Fidelix MSP. Manual Orientativo: Sistematização do Cuidado de Nutrição / [organizado pela] Associação Brasileira de Nutrição. São Paulo: Associação Brasileira de Nutrição; 2014.

11. WHO. World Health Organization. Global report on falls prevention in older age. 2007 [cited 2015 Jan 13]. Available from: http://www.who.int/ageing/publications/ Falls_prevention7March.pdf

12. Souza S et al. Desnutrição, quedas e necessidade de cuidador em idosos que beneficiam de um apoio geriatric. APNEP. 2012 jul.

13. Queiroz L, Lira S, Sasaki A. Identificação do risco de quedas pela avaliação da mobilidade funcional em idosos hospitalizados. Rev. Baiana de Saude Publica. 2009; 33(4):534-43.

14. Guigoz Y, Vellas B, Garry P. Mini nutritional assessment: a practical assessment tool for grading the nutritional state of elderly patients. Facts, Res Gerontol. 1994;4(Suppl. 2): $15 \mathrm{e} 59$.

15. Guigoz Y. The mini nutritional assessment (MNA) review of the literature-what dose it tell us? J Nutr Health Aging. 2006; 10:466e 85 .

16.Urbanetto JS, et al. Morse Fall Scale: tradução e adaptação transcultural para a língua portuguesa. Rev Esc Enferm USP. 2013 June;47(3):569-75.

17. Nestle Nutrition Institute [internet] MNA Mini Nutritional Assessment [cited 2015 Dec 17]. Available from: http://www. mna-elderly.com/identifying malnutrition.html

18. Silva JL, Marques APO. Idosos institucionalizados: um estudo sobre o risco nutricional e seus fatores associados. (mestrado) Recife: Universidade Federal de Pernambuco; 2014.

19. Duarte JP, et al. Variação na prevalência de risco nutricional em indivíduos hospitalizados conforme cinco protocolos de triagem nutricional. Rev Scientia Medica. 2014;24(1):26-32.

20. Morais FTD, Campos IC, Lessa NMV. Diagnóstico Nutricional em idosos hospitalizados. Nutrir Gerais. 2010; 4(7):637-51.

21. Rocha HB, et al Avaliação do risco de quedas em adultos hospitalizados conforme a morse fall scale traduzida para a língua portuguesa. Rev Graduação. 2013;6 (1).

22. Bailey PH, et al. Description of a process to calibrate the Morse fall scale in a long-term care home. Appl Nurs Res. 2011 Nov;24(4):263-8

23. Ming-Hung Chien, How-Ran Guo Nutritional Status and Falls in Community-Dwelling Older People: A Longitudinal Study of a Population-Based Random Sample. PLoS One. 2014 Mar 10;9(3):e91044.

24. Neyens J, Halfens R, Spreeuwenberg M, Meijers J, Luiking $\mathrm{Y}$, Schols J. Malnutrition is associated with an increased risk os falls and impaired activity in elderly patients in Dutch residential long-term care: a cross-sectional study. Arch Gerontol Geriatr 2013;56(1):265-9. 